\title{
Estimação Semicega de Canais com Correlação Usando Filtro de Kalman e Códigos Espaço-Temporais
}

\author{
Murilo Bellezoni Loiola e Renato da Rocha Lopes
}

\begin{abstract}
Resumo-Neste artigo propomos um algoritmo semicego para estimação de canais MIMO planos, variantes no tempo e com correlação espacial entre as antenas. $O$ estimador proposto utiliza um filtro de Kalman para rastrear o canal em sistemas empregando códigos espaço-temporais ortogonais por blocos. Após estabelecer um modelo em espaço de estados do canal com correlação espacial, mostramos que o estimador proposto pode ser simplificado graças à natureza ortogonal das palavrascódigo espaço-temporais. Resultados de simulações indicam que o desempenho do algoritmo proposto não depende da correlação espacial entre as antenas.
\end{abstract}

Palavras-Chave-Estimação de canais MIMO, filtro de Kalman, códigos espaço-temporais ortogonais por blocos, correlação espacial.

Abstract - In this paper we propose a semiblind algorithm for estimation of flat, time-varying and spatially correlated MIMO channels. The proposed estimator employs a Kalman filter to track the channel in orthogonal space-time block coded systems. After developing a state-space model to spatially correlated MIMO channels we show that the proposed estimator can be simplified by using the orthogonality inherent to space-time block codewords. Simulation results indicate that the proposed algorithm is robust to antennas spatial correlations.

Keywords-MIMO channel estimation, Kalman filter, orthogonal space-time block codes, spatial correlation.

\section{INTRODUÇÃO}

Uma maneira efetiva e prática de se explorar diversidade em sistemas com múltiplas entradas e múltiplas saídas (MIMO, do inglês Multiple Input, Multiple Output) é empregar códigos espaço-temporais. Como o próprio nome sugere, a codificação dos códigos espaço-temporais é feita nos domínios espacial e temporal, introduzindo correlação entre os sinais transmitidos por diferentes antenas em diferentes intervalos de tempo. Esta correlação espaço-temporal entre os sinais transmitidos é utilizada para reduzir os efeitos do desvanecimento do canal e melhorar a qualidade do sinal recebido. $O$ uso da codificação espaço-temporal possibilita a obtenção de diversidade de transmissão sem aumento da potência total do sistema.

Dentre os diferentes esquemas de codificação espaçotemporal existentes, os códigos espaço-temporais ortogonais por blocos (OSTBC, do inglês Orthogonal Space-Time Block

Murilo Bellezoni Loiola e Renato da Rocha Lopes, Laboratório de Processamento de Sinais para Comunicações, Departamento de Comunicações, Faculdade de Engenharia Elétrica e de Computação, Universidade Estadual de Campinas, Campinas, Brasil, e-mails: \{mloiola, rlopes\}@decom.fee.unicamp.br. Este trabalho foi financiado pela FAPESP (05/55310-8) e pelo CNPq (311844/2006-5).
Codes) [1], [2] são particularmente interessantes pois permitem alcançar diversidade máxima usando receptores de baixa complexidade. Mais precisamente, o receptor de máxima verossimilhança (MV) para OSTBC's consiste em um simples receptor linear seguido por um decisor.

Para decodificar corretamente os sinais recebidos, o receptor MV deve ter conhecimento perfeito do canal. Como normalmente os receptores não dispõem de tal informação, técnicas de estimação de canal são essenciais para o bom funcionamento do sistema. Quando o canal é estático, métodos como os apresentados em [3] e [4] podem ser empregados com sucesso. Porém, na prática, o canal pode variar com o tempo em decorrência, por exemplo, da mobilidade do transmissor e/ou do receptor. Neste caso, o algoritmo de estimação deve ser capaz de acompanhar as variações do canal. Um dos algoritmos adaptativos mais empregados para tal fim é o filtro de Kalman (FK) [5], graças à sua habilidade intrínseca de funcionamento em ambientes não-estacionários. Em [6], um FK foi usado para estimar canais MIMO planos, com desvanecimento rápido, em sistemas com codificação espaço-temporal baseada no código de Alamouti [1]. Já o estimador semicego proposto em [7] generaliza o método proposto em [6] para qualquer tipo de OSTBC.

Uma hipótese fundamental no desenvolvimento dos algoritmos propostos em [6] e [7] é a independência entre os coeficientes do canal de comunicação. Porém, em situações reais, pode existir uma correlação espacial entre as antenas de transmissão e/ou de recepção. A presença de correlação espacial entre as antenas degrada o desempenho dos OSTBC bem como reduz os ganhos de capacidade dos canais MIMO. Portanto, neste artigo propomos um estimador, baseado no filtro de Kalman, para canais MIMO planos, variantes no tempo e que apresentam correlação espacial entre as antenas transmissoras e/ou receptoras. Graças ao uso de OSTBC's, o filtro de Kalman original pode ser simplificado, levando a um algoritmo de complexidade reduzida. Os algoritmos propostos em [6] e [7] podem, portanto, ser considerados como casos particulares do algoritmo exposto neste trabalho.

O restante deste artigo está organizado da seguinte maneira: a seção II apresenta o sistema estudado, descrevendo brevemente alguns modelos de canal e as características principais dos códigos espaço-temporais ortogonais por blocos. Já a seção III mostra em detalhes a derivação do algoritmo de complexidade reduzida proposto no presente trabalho, enquanto que os resultados de simulações são apresentados na seção IV. Por fim, na seção V estão contidas as conclusões. 


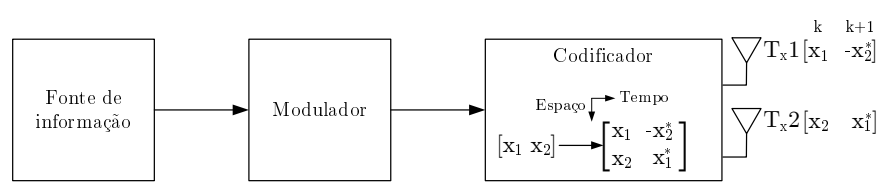

Fig. 1. Diagrama de blocos do codificador espaço-temporal de Alamouti.

\section{Modelo de Sistema}

Considere um sistema MIMO com $N_{T}$ antenas transmissoras enviando blocos de dados de tamanho $T$ para $N_{R}$ antenas receptoras. O canal é suposto plano e constante durante a transmissão de um bloco de dados, podendo variar entre blocos consecutivos. A relação entre os sinais transmitido e recebido para o bloco $k$ pode ser expressa por

$$
\mathbf{Y}_{k}=\mathbf{H}_{k} \mathbf{X}_{k}+\mathbf{N}_{k}
$$

onde $\mathbf{Y}_{k}$ é uma matriz $N_{R} \times T$ com os sinais recebidos, $\mathbf{X}_{k}$ é uma matriz $N_{T} \times T$ contendo os sinais transmitidos, $\mathbf{N}_{k}$, de dimensões $N_{R} \times T$, contém amostras independentes de ruído gaussiano, branco, circularmente simétrico, de média nula e matriz de covariância $\mathbf{R}_{n}=\sigma_{n}^{2} \mathbf{I}$, enquanto que o canal é representado pela matriz $\mathbf{H}_{k}$, de tamanho $N_{R} \times N_{T}$.

$\mathrm{Na}$ codificação espaço-temporal por blocos, a matriz $\mathbf{X}_{k}$ representa um mapeamento que transforma um bloco de símbolos de comprimento $K, \mathbf{x}_{k}=\left[\begin{array}{llll}x_{k}^{(1)} & x_{k}^{(2)} & \cdots & x_{k}^{(K)}\end{array}\right]^{\mathrm{T}}$, onde $(\cdot)^{\mathrm{T}}$ indica transposição, em uma matriz $N_{T} \times T$. A matriz $\mathbf{X}_{k}$ é dita um OSTBC se [1]-[3]: 1) os elementos de $\mathbf{X}_{k}$ forem funções lineares de todos os símbolos de $\mathbf{x}_{k}$ e seus complexos conjugados; 2) para um $\mathbf{x}_{k}$ arbitrário, $\mathbf{X}_{k} \mathbf{X}_{k}^{\mathrm{H}}=$ $\left\|\mathbf{x}_{k}\right\|^{2} \mathbf{I}_{N_{T}}$, onde $\mathbf{I}_{N_{T}}$ representa a matriz identidade de ordem $N_{T},\|\cdot\|^{2}$ é o quadrado da norma euclidiana e $(\cdot)^{\mathrm{H}}$ denota o conjugado transposto de uma matriz.

O primeiro OSTBC proposto foi apresentado por Alamouti em [1]. Para este código, a palavra-código espaço-temporal $\mathbf{X}_{k}$ é dada por

$$
\mathbf{X}_{k}=\left[\begin{array}{cc}
x_{k}^{(1)} & -\left(x_{k}^{(2)}\right)^{*} \\
x_{k}^{(2)} & \left(x_{k}^{(1)}\right)^{*}
\end{array}\right]
$$

com $\mathbf{x}_{k}=\left[\begin{array}{ll}x_{k}^{(1)} & x_{k}^{(2)}\end{array}\right]^{\mathrm{T}}$ e $(\cdot)^{*}$ representando o complexo conjugado. A Fig. 1 apresenta o diagrama de blocos do codificador espaço-temporal de Alamouti.

O código de Alamouti utiliza duas antenas transmissoras e dois períodos de símbolo para atingir diversidade total com um código de taxa 1. No primeiro uso do canal, os símbolos $x_{k}^{(1)}$ e $x_{k}^{(2)}$ são transmitidos, respectivamente, pelas antenas transmissoras 1 e 2 . Já no segundo uso do canal, $-\left(x_{k}^{(2)}\right)^{*}$ é enviado pela antena um, enquanto $\left(x_{k}^{(1)}\right)^{*}$ é enviado pela antena 2 .

Uma das principais características de qualquer OSTBC é a simplicidade do decodificador. Mais precisamente, o receptor de MV para OSTBC's consiste em um simples processamento linear seguido por um decisor. $\mathrm{O}$ processamento linear gera estatísticas de decisão através de uma combinação linear do sinais recebidos. Estas estatísticas de decisão são, então, passadas ao decisor. Para o código de Alamouti, as estatísticas de decisão são calculadas como

$$
\begin{aligned}
& \tilde{x}_{k}^{(1)}=\sum_{i=1}^{N_{R}}\left(h_{k}^{(i, 1)}\right)^{*} y_{1}^{(i)}+h_{k}^{(i, 2)}\left(y_{2}^{(i)}\right)^{*} \\
& \tilde{x}_{k}^{(2)}=\sum_{i=1}^{N_{R}}\left(h_{k}^{(i, 2)}\right)^{*} y_{1}^{(i)}-h_{k}^{(i, 1)}\left(y_{2}^{(i)}\right)^{*},
\end{aligned}
$$

onde $y_{m}^{(i)}, m=1,2$, corresponde ao sinal recebido pela antena receptora $i$ no $m$-ésimo intervalo de transmissão do bloco $k$ e $h_{k}^{(i, j)}, \ldots, N_{R}, j=1, \ldots, N_{T}$ representa o elemento $(i, j)$ da matriz $\mathbf{H}_{k}$.

Como mencionado anteriormente, o canal é suposto invariante durante a transmissão da palavra-código espaço-temporal $k$, porém variante entre palavras-código diferentes. De acordo com o amplamente utilizado modelo estacionário no sentido amplo com espalhadores descorrelacionados (WSSUS, do inglês Wide-Sense Stationary Uncorrelated Scattering) [8], os coeficientes do canal podem ser modelados como variáveis aleatórias independentes, gaussianas, complexas, de média zeros e função de autocorrelação temporal dada por [9], [10]

$$
\mathrm{E}\left[h_{k}^{(i, j)}\left(h_{t}^{(i, j)}\right)^{*}\right] \approx \mathcal{J}_{0}\left(2 \pi f_{D} T|k-t|\right),
$$

onde $\mathcal{J}_{0}$ é a função de Bessel do primeiro tipo e ordem zero, $f_{D} T$ é o desvio Doppler normalizado (considerado igual para todos os pares de antenas transmissoras-receptoras) e $T$ é a taxa de símbolo do sistema. Logo, o espectro normalizado para cada coeficiente do canal é expresso por

$$
S(f)=\left\{\begin{array}{cl}
\frac{1}{\pi f_{D} T} \frac{1}{\sqrt{1-\left(\frac{f}{f_{D} T}\right)^{2}}}, & |f|<f_{D} T \\
0, & \text { caso contrário. }
\end{array}\right.
$$

Embora a representação exata da dinâmica do canal por um processo auto-regressivo (AR) de ordem finita seja impossível uma vez que a função de autocorrelação temporal (4) não é racional e o espectro (5) é de banda limitada, é possível aproximar a evolução temporal dos coeficientes do canal por processos $\mathrm{AR}$ de ordem pequena. Isto porque os primeiros termos de (4), para atraso pequenos, capturam a maior parte da dinâmica do canal [9]. Conseqüentemente, assim como em [9] e [10], aproximaremos as variações do canal MIMO por um processo AR de primeira ordem. Portanto, a evolução temporal dos coeficientes independentes do canal é dada por

$$
\mathbf{h}_{k}^{\text {ind }}=\mathbf{F h}_{k-1}^{\text {ind }}+\mathbf{w}_{k},
$$

onde $\mathbf{h}_{k}=\operatorname{vec}\left(\mathbf{H}_{k}\right)$ é o vetor obtido pelo empilhamento das colunas da matriz $\mathbf{H}_{k}$, $\operatorname{vec}(\cdot)$ denota o operador responsável pelo empilhamento das colunas de uma matriz e o índice "ind" indica que os coeficientes do canal são espacialmente independentes. Além disso, tem-se que

$$
\mathbf{F}=\beta \mathbf{I}_{N_{R} N_{T}},
$$

$\beta=\mathcal{J}_{0}\left(2 \pi f_{D} T\right), \mathbf{w}_{k}$ é um vetor de tamanho $N_{R} N_{T}$ contendo amostras independentes do ruído de excitação gaussiano, circularmente simétrico, de média zero e matriz de covariância $\mathbf{Q}=\sigma_{w}^{2} \mathbf{I}_{N_{R} N_{T}}, \sigma_{w}^{2}=\left(1-|\beta|^{2}\right) P_{k}$ e $P_{k}=\mathrm{E}\left[\left|h_{k}^{(m)}\right|^{2}\right]$, $m=1, \ldots, N_{R} N_{T}$. 
A velocidade das variações do canal, quantificada por $\beta$ em (7), é determinada pelo desvio Doppler ou, equivalentemente, pela velocidade relativa entre as $N_{T}$ antenas transmissoras e as $N_{R}$ antenas receptoras. Quanto maior o valor de $f_{D} T$, menor o valor de $\beta$ e, conseqüentemente, mais rápidas as variações do canal. A magnitude destas variações é controlada por $\sigma_{w}$.

Em situações reais, contudo, os coeficientes do canal podem não ser totalmente independentes, apresentando uma correlação espacial devida, por exemplo, a uma pequena separação entre as antenas transmissoras. Um dos modelos mais utilizados quando existe correlação espacial entre as antenas é o modelo de Kronecker [11], dado por

$$
\mathbf{H}_{k}^{\text {cor }}=\mathbf{R}_{R x}^{1 / 2} \mathbf{H}_{k}^{\text {ind }}\left(\mathbf{R}_{T x}^{1 / 2}\right)^{\mathrm{T}}
$$

onde $\mathbf{R}_{R x}$ e $\mathbf{R}_{T x}$ são, respectivamente, as matrizes de correlação espacial entre as antenas de recepção e de transmissão e $\mathbf{H}_{k}^{\text {ind }}$ representa o canal com coeficientes independentes.

Como o modelo (8) pode ser obtido a partir do modelo com coeficientes descorrelacionados, seria natural tentar formular a evolução temporal do canal com correlação espacial de forma semelhante a (6). Em [12], um modelo AR de primeira ordem para canais com correlação espacial é desenvolvido usando um modelo diferente do mostrado em (8). Infelizmente, os autores de [12] não explicitam a forma da matriz de transição de estados do modelo AR, dizendo apenas que ela é estimada a partir de medidas de canais reais. Desta maneira, é necessário desenvolver um modelo para caracterizar a evolução temporal do canal com correlação espacial. Isto é feito na próxima subseção.

\section{A. Modelo de canal correlacionado variante no tempo}

Antes de modelar a dinâmica do canal MIMO com correlação espacial, é importante lembrar que, para três matrizes quaisquer $\mathbf{M}, \mathbf{S}$ e $\mathbf{J}$ de dimensões compatíveis, podemos reescrever $\mathbf{Z}=\mathbf{J M S}$ como [13]

$$
\operatorname{vec}(\mathbf{Z})=\left(\mathbf{S}^{\mathrm{T}} \otimes \mathbf{J}\right) \operatorname{vec}(\mathbf{M}) \text {, }
$$

onde $\otimes$ representa o produto de Kronecker.

Assim, empilhando as colunas de $\mathbf{H}_{k}^{\text {cor }}$ em (8) e usando (9), obtém-se

$$
\operatorname{vec}\left(\mathbf{H}_{k}^{\text {cor }}\right)=\mathbf{h}_{k}^{\text {cor }}=\left(\mathbf{R}_{T x}^{1 / 2} \otimes \mathbf{R}_{R x}^{1 / 2}\right) \operatorname{vec}\left(\mathbf{H}_{k}^{\text {ind }}\right)=\mathbf{G h}_{k}^{\text {ind }},
$$

onde definiu-se $\mathbf{G}=\left(\mathbf{R}_{T x}^{1 / 2} \otimes \mathbf{R}_{R x}^{1 / 2}\right)$.

Podemos também definir a matriz $\mathbf{R}_{h}$ como

$$
\begin{aligned}
\mathbf{R}_{h} & =\mathbf{G G}^{\mathrm{H}}=\left(\mathbf{R}_{T x}^{1 / 2} \otimes \mathbf{R}_{R x}^{1 / 2}\right)\left(\mathbf{R}_{T x}^{1 / 2} \otimes \mathbf{R}_{R x}^{1 / 2}\right)^{\mathrm{H}} \\
& =\left(\mathbf{R}_{T x}^{1 / 2} \otimes \mathbf{R}_{R x}^{1 / 2}\right)\left(\mathbf{R}_{T x}^{\mathrm{H} / 2} \otimes \mathbf{R}_{R x}^{\mathrm{H} / 2}\right) \\
& =\left(\mathbf{R}_{T x}^{1 / 2} \mathbf{R}_{T x}^{\mathrm{H} / 2} \otimes \mathbf{R}_{R x}^{1 / 2} \mathbf{R}_{R x}^{\mathrm{H} / 2}\right)=\left(\mathbf{R}_{T x} \otimes \mathbf{R}_{R x}\right) .
\end{aligned}
$$

Pré-multiplicando (6) por G, obtém-se

$$
\mathbf{G h}_{k}^{\text {ind }}=\mathbf{G} \mathbf{F} \mathbf{h}_{k-1}^{\text {ind }}+\mathbf{G w}_{k}=\beta \mathbf{G h}_{k-1}^{\text {ind }}+\mathbf{G w}_{k},
$$

onde usamos o fato de a matriz de transição de estados $\mathbf{F}$ ser diagonal. Desta maneira, comparando (12) e (10), é possível escrever a representação em espaço de estados do canal MIMO variante no tempo com correlação espacial como

$$
\mathbf{h}_{k}^{\text {cor }}=\beta \mathbf{h}_{k-1}^{\text {cor }}+\mathbf{G w}_{k} \text {. }
$$

\section{RECEPTOR PROPOSTO}

Procedendo agora ao empilhamento das colunas de $\mathbf{Y}_{k}, \mathbf{H}_{k}$ e $\mathbf{N}_{k}$ em (1), obtemos

$$
\mathbf{y}_{k}=\mathcal{X}_{k} \mathbf{h}_{k}+\mathbf{n}_{k},
$$

onde $\mathcal{X}_{k}=\mathbf{X}_{k}^{\mathrm{T}} \otimes \mathbf{I}_{N_{R}}$ foi calculado usando (9).

Como mencionado na seção II, uma das características dos OSTBC's é a ortogonalidade das palavra-códigos. Esta propriedade se mantém na nova palavra-código $\mathcal{X}_{k}$ uma vez que

$$
\begin{aligned}
\mathcal{X}_{k}^{\mathrm{H}} \mathcal{X}_{k} & =\left(\mathbf{X}_{k}^{\mathrm{T}} \otimes \mathbf{I}_{N_{R}}\right)^{\mathrm{H}}\left(\mathbf{X}_{k}^{\mathrm{T}} \otimes \mathbf{I}_{N_{R}}\right) \\
& =\left(\mathbf{X}_{k}^{*} \otimes \mathbf{I}_{N_{R}}\right)\left(\mathbf{X}_{k}^{\mathrm{T}} \otimes \mathbf{I}_{N_{R}}\right)=\mathbf{X}_{k}^{*} \mathbf{X}_{k}^{\mathrm{T}} \otimes \mathbf{I}_{N_{R}} \\
& =\left(\mathbf{X}_{k} \mathbf{X}_{k}^{\mathrm{H}}\right)^{\mathrm{T}} \otimes \mathbf{I}_{N_{R}}=\left\|\mathbf{x}_{k}\right\|^{2} \mathbf{I}_{N_{T}} \otimes \mathbf{I}_{N_{R}} \\
& =\left\|\mathbf{x}_{k}\right\|^{2} \mathbf{I}_{N_{R} N_{T}},
\end{aligned}
$$

sendo $\mathbf{X}_{k}^{*}$ o complexo conjugado da matriz $\mathbf{X}_{k}$ e $\mathbf{X}_{k} \mathbf{X}_{k}^{\mathrm{H}}=$ $\left\|\mathbf{x}_{k}\right\|^{2} \mathbf{I}_{N_{T}}$.

Por fim, reunindo (13) e (14), obtemos a formulação em espaço de estados do problema de estimação da canais MIMO planos, variantes no tempo e com correlação espacial entre as antenas:

$$
\begin{array}{rlr}
\mathbf{h}_{k}^{\text {cor }} & =\beta \mathbf{h}_{k-1}^{\text {cor }}+\mathbf{G w}_{k} & \text { (Eq. processo) } \\
\mathbf{y}_{k} & =\mathcal{X}_{k} \mathbf{h}_{k}^{\text {cor }}+\mathbf{n}_{k} & \text { (Eq. observação) } .
\end{array}
$$

Como tanto (16a) quanto (16b) são funções lineares das variáveis de estado $\mathbf{h}_{k}^{\text {cor }}$ e os ruídos $\mathbf{w}_{k}$ e $\mathbf{n}_{k}$ são gaussianos, brancos e independentes, o filtro de Kalman fornece o estimador recursivo ótimo (no sentido de minimização do erro quadrático médio de estimação) dos coeficientes do canal [5]. Desta maneira, o algoritmo no quadro 1 apresenta o estimador de canal proposto baseado no filtro de Kalman.

$$
\begin{aligned}
& \text { Quadro } 1 \text { Filtro de Kalman } \\
& \text { Predição } \\
& \begin{array}{c}
\hat{\mathbf{h}}_{k \mid k-1}=\mathbf{F} \hat{\mathbf{h}}_{k-1 \mid k-1} \\
\mathbf{P}_{k \mid k-1}=\mathbf{F P}_{k-1 \mid k-1} \mathbf{F}^{\mathrm{H}}+\mathbf{G Q G}^{\mathrm{H}}
\end{array} \\
& \mathbf{K}_{k}=\mathbf{P}_{k \mid k-1} \mathcal{X}_{k}^{\mathrm{H}}\left[\mathcal{X}_{k} \mathbf{P}_{k \mid k-1} \mathcal{X}_{k}^{\mathrm{H}}+\mathbf{R}_{n}\right]^{-1} \\
& \alpha_{k}=\mathbf{y}_{k}-\mathcal{X}_{k} \hat{\mathbf{h}}_{k \mid k-1} \\
& \hat{\mathbf{h}}_{k \mid k}=\hat{\mathbf{h}}_{k \mid k-1}+\mathbf{K}_{k} \alpha_{k} \\
& \mathbf{P}_{k \mid k}=\left[\mathbf{I}-\mathbf{K}_{k} \mathcal{X}_{k}\right] \mathbf{P}_{k \mid k-1}
\end{aligned}
$$

Nestas equações, $\mathbf{P}_{k \mid k-1}$ é a matriz de correlação do erro de predição de estados e $\hat{\mathbf{h}}_{k \mid k-1}$ é o vetor contendo os valores preditos dos estados, num instante $k$, obtidos a partir dos sinais observados do instante inicial até o instante $k-1$. 
Para reduzir a complexidade computacional do algoritmo do quadro 1, podemos utilizar as propriedades dos OSTBC's bem como as características do sistema. Assim, substituindo (7) em (17a), temos

$$
\hat{\mathbf{h}}_{k \mid k-1}=\mathbf{F} \hat{\mathbf{h}}_{k-1 \mid k-1}=\beta \mathbf{I}_{N_{R} N_{T}} \hat{\mathbf{h}}_{k-1 \mid k-1}=\beta \hat{\mathbf{h}}_{k-1 \mid k-1} .
$$

Usando agora (7) e (11) em (17b), obtemos

$$
\begin{aligned}
\mathbf{P}_{k \mid k-1} & =\mathbf{F P}_{k-1 \mid k-1} \mathbf{F}^{\mathrm{H}}+\mathbf{G Q G}^{\mathrm{H}} \\
& =\beta^{2} \mathbf{P}_{k-1 \mid k-1}+\sigma_{w}^{2} \mathbf{G G}^{\mathrm{H}}=\beta^{2} \mathbf{P}_{k-1 \mid k-1}+\sigma_{w}^{2} \mathbf{R}_{h} .
\end{aligned}
$$

Utilizando o lema de inversão de matrizes juntamente com (15) e lembrando que $\mathbf{R}_{n}=\sigma_{n}^{2} \mathbf{I}_{N_{R} N_{T}}$, podemos reescrever (18a) como

$$
\begin{aligned}
& \mathbf{K}_{k}= \\
& \mathbf{P}_{k \mid k-1} \mathcal{X}_{k}^{\mathrm{H}}\left[\mathbf{R}_{n}^{-1}-\mathbf{R}_{n}^{-1} \mathcal{X}_{k}\left(\mathcal{X}_{k}^{\mathrm{H}} \mathbf{R}_{n}^{-1} \mathcal{X}_{k}+\mathbf{P}_{k \mid k-1}^{-1}\right)^{-1} \mathcal{X}_{k}^{\mathrm{H}} \mathbf{R}_{n}^{-1}\right] \\
& =\mathbf{P}_{k \mid k-1} \mathcal{X}_{k}^{\mathrm{H}}\left[\frac{1}{\sigma_{n}^{2}} \mathbf{I}_{N_{R} N_{T}}-\frac{1}{\sigma_{n}^{4}} \mathcal{X}_{k}\left(\frac{1}{\sigma_{n}^{2}} \mathcal{X}_{k}^{\mathrm{H}} \mathcal{X}_{k}+\mathbf{P}_{k \mid k-1}^{-1}\right)^{-1} \mathcal{X}_{k}^{\mathrm{H}}\right] \\
& =\frac{1}{\sigma_{n}^{2}} \mathbf{P}_{k \mid k-1}\left[\mathcal{X}_{k}^{\mathrm{H}}-\frac{\left\|\mathbf{x}_{k}\right\|^{2}}{\sigma_{n}^{2}}\left(\frac{\left\|\mathbf{x}_{k}\right\|^{2}}{\sigma_{n}^{2}} \mathbf{I}_{N_{R} N_{T}}+\mathbf{P}_{k \mid k-1}^{-1}\right)^{-1} \mathcal{X}_{k}^{\mathrm{H}}\right] \\
& =\mathbf{A}_{k} \mathcal{X}_{k}^{\mathrm{H}},
\end{aligned}
$$

onde definiu-se

$\mathbf{A}_{k}=\frac{1}{\sigma_{n}^{2}} \mathbf{P}_{k \mid k-1}\left[\mathbf{I}_{N_{R} N_{T}}-\frac{\left\|\mathbf{x}_{k}\right\|^{2}}{\sigma_{n}^{2}}\left(\frac{\left\|\mathbf{x}_{k}\right\|^{2}}{\sigma_{n}^{2}} \mathbf{I}_{N_{R} N_{T}}+\mathbf{P}_{k \mid k-1}^{-1}\right)^{-1}\right]$

Substituindo (21) em (18c), temos

$$
\begin{aligned}
\hat{\mathbf{h}}_{k \mid k} & =\hat{\mathbf{h}}_{k \mid k-1}+\mathbf{K}_{k}\left(\mathbf{y}_{k}-\mathcal{X}_{k} \hat{\mathbf{h}}_{k \mid k-1}\right) \\
& =\hat{\mathbf{h}}_{k \mid k-1}+\mathbf{A}_{k} \mathcal{X}_{k}^{\mathrm{H}}\left(\mathbf{y}_{k}-\mathcal{X}_{k} \hat{\mathbf{h}}_{k \mid k-1}\right) \\
& =\left(\mathbf{I}_{N_{R} N_{T}}-\left\|\mathbf{x}_{k}\right\|^{2} \mathbf{A}_{k}\right) \hat{\mathbf{h}}_{k \mid k-1}+\mathbf{A}_{k} \mathcal{X}_{k}^{\mathrm{H}} \mathbf{y}_{k} \\
& =\beta \mathbf{B}_{k} \hat{\mathbf{h}}_{k-1 \mid k-1}+\mathbf{A}_{k} \mathcal{X}_{k}^{\mathrm{H}} \mathbf{y}_{k},
\end{aligned}
$$

sendo $\mathbf{B}_{k}=\left(\mathbf{I}_{N_{R} N_{T}}-\left\|\mathbf{x}_{k}\right\|^{2} \mathbf{A}_{k}\right)$.

Finalmente, substituindo (21) em (18d), obtemos

$$
\begin{aligned}
\mathbf{P}_{k \mid k} & =\left[\mathbf{I}_{N_{R} N_{T}}-\mathbf{K}_{k} \mathcal{X}_{k}\right] \mathbf{P}_{k \mid k-1}=\left[\mathbf{I}_{N_{R} N_{T}}-\mathbf{A}_{k} \mathcal{X}_{k}^{\mathrm{H}} \mathcal{X}_{k}\right] \mathbf{P}_{k \mid k-1} \\
& =\left[\mathbf{I}_{N_{R} N_{T}}-\left\|\mathbf{x}_{k}\right\|^{2} \mathbf{A}_{k}\right] \mathbf{P}_{k \mid k-1}=\mathbf{B}_{k} \mathbf{P}_{k \mid k-1} .
\end{aligned}
$$

O algoritmo do filtro de Kalman com complexidade reduzida pode ser visto no quadro 2 .

Quadro 2 Filtro de Kalman com complexidade reduzida

$$
\begin{aligned}
& \mathbf{P}_{k \mid k-1}=\beta^{2} \mathbf{P}_{k-1 \mid k-1}+\sigma_{w}^{2} \mathbf{R}_{h} \\
& \mathbf{A}_{k}=\frac{1}{\sigma_{n}^{2}} \mathbf{P}_{k \mid k-1}\left[\mathbf{I}_{N_{R} N_{T}}-\frac{\left\|\mathbf{x}_{k}\right\|^{2}}{\sigma_{n}^{2}}\left(\frac{\left\|\mathbf{x}_{k}\right\|^{2}}{\sigma_{n}^{2}} \mathbf{I}_{N_{R} N_{T}}-\mathbf{P}_{k \mid k-1}^{-1}\right)^{-1}\right] \\
& \mathbf{B}_{k}=\mathbf{I}_{N_{R} N_{T}}-\left\|\mathbf{x}_{k}\right\|^{2} \mathbf{A}_{k} \\
& \hat{\mathbf{h}}_{k \mid k}=\beta \mathbf{B}_{k} \hat{\mathbf{h}}_{k-1 \mid k-1}+\mathbf{A}_{k} \mathcal{X}_{k}^{\mathrm{H}} \mathbf{y}_{k} \\
& \mathbf{P}_{k \mid k}=\mathbf{B}_{k} \mathbf{P}_{k \mid k-1}
\end{aligned}
$$

Considerando que seqüências de treinamento são inseridas periodicamente entre os símbolos de informação, o algoritmo proposto no quadro 2 pode funcionar em modo semicego. Primeiramente, quando seqüências de treinamento estão disponíveis, a matriz $\mathcal{X}_{k}$ em (23) é construída a partir dos símbolos conhecidos pelo receptor. Finalizada a transmissão dos símbolos de treinamento, o algoritmo passa para o modo de realimentação de decisão, sendo a matriz $\mathcal{X}_{k}$ gerada com as estimativas obtidas pelo decodificador de máxima verossimilhança dos OSTBC's usando as estimativas de canal fornecidas pelo filtro de Kalman. Para o código de Alamouti, por exemplo, o FK operando em modo de realimentação de decisão calcularia as estatísticas de decisão (3) para fazer a decodificação MV dos sinais recebidos após o cálculo da matriz $\mathbf{B}_{k}$ e antes da equação de atualização de $\hat{\mathbf{h}}_{k \mid k}$.

\section{SimulaçÕES}

Nesta seção, alguns resultados de simulação serão apresentados para ilustrar o desempenho do estimador semi-cego proposto no presente trabalho.

Nas simulações realizadas foi considerado um sistema MIMO com duas antenas de transmissão $\left(N_{T}=2\right)$ enviando símbolos QPSK para duas antenas de recepção $\left(N_{R}=2\right)$. Antes da transmissão, os símbolos foram codificados usando o código espaço-temporal de Alamouti. A cada 250 blocos de informação foram inseridos 25 blocos de treinamento. Foram simulados canais com freqüências Doppler normalizadas iguais a $f_{D} T=0,001$ e $f_{D} T=0,007$. Para uma taxa de $144 \mathrm{~kb} / \mathrm{s}$ e freqüência da portadora em $1,9 \mathrm{GHz}$, os valores de $f_{D} T$ considerados correspondem a terminais móveis de deslocando, respectivamente, a 41 e $286 \mathrm{~km} / \mathrm{h}$. Para cada valor de $f_{D} T$, foram simulados diferentes valores de correlação espacial, $p_{T x}$, entre as antenas de transmissão. A matriz $\mathbf{R}_{T_{x}}$ considerada tem a seguinte forma:

$$
\mathbf{R}_{T x}=\left[\begin{array}{cc}
1 & p_{T x} \\
p_{T x} & 1
\end{array}\right] .
$$

Supomos também que as antenas de recepção não possuem correlação espacial e que o receptor conhece os valores de $\beta$ e das variâncias dos ruídos envolvidos na formulação em espaço de estados. Os resultados a seguir representam a média de dez realizações diferentes do canal.

A Fig. 2 mostra as taxas de erro de símbolos para o receptor com conhecimento perfeito de canal e para o receptor semicego proposto, com $f_{D} T=0,001$. Como era esperado, a existência de correlação espacial no canal degrada o desempenho dos OSTBC's. Para uma taxa de erro de $10^{-3}$, por exemplo, o sistema com $p_{T_{x}}=0,7$ fica a cerca de $3 \mathrm{~dB}$ do desempenho do sistema sem correlação espacial. Podemos ainda notar que, para os dois valores de $p_{T_{x}}$ simulados $\left(p_{T_{x}}=0\right.$ e $\left.p_{T_{x}}=0,7\right)$, o receptor proposto apresentou desempenho equivalente ao do receptor com conhecimento perfeito do canal, indicando que o FK proposto possui uma boa capacidade de rastreamento. $\mathrm{O}$ erro quadrático médio de estimação do canal pode ser visto na Fig. 3. É interessante observar que o desempenho do algoritmo de estimação de canal não é afetado pela correlação espacial entre as antenas de transmissão. Isto indica que o FK 


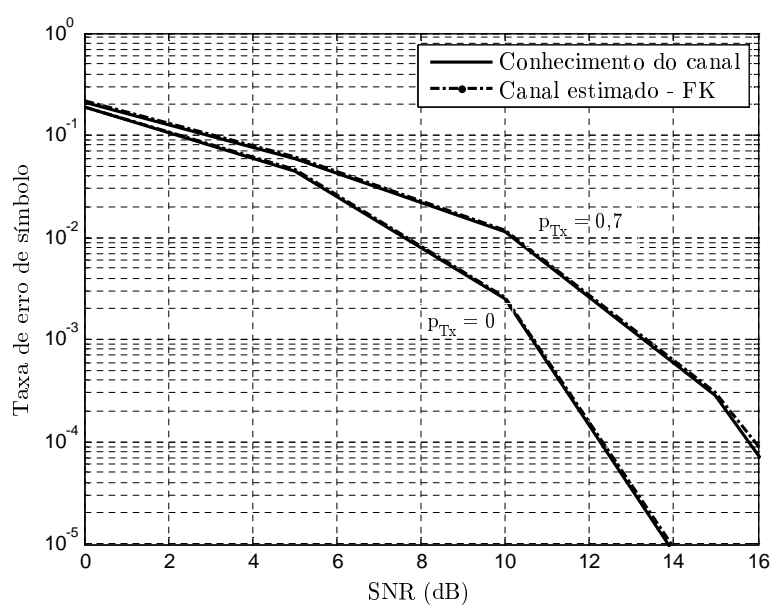

Fig. 2. Taxa de erro de símbolos para $f_{D} T=0,001$.

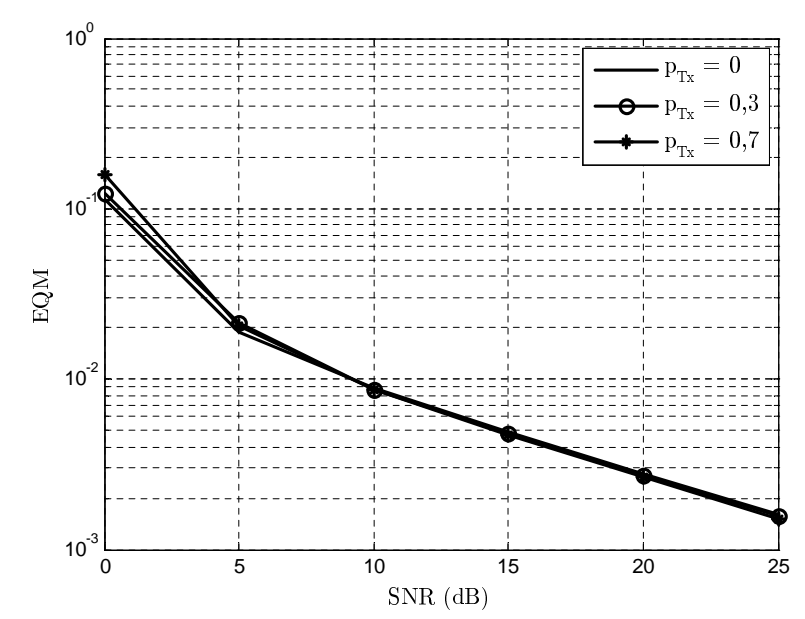

Fig. 3. Erro quadrático médio de estimação para $f_{D} T=0,001$.

desenvolvido é robusto em relação à correlação espacial do canal.

Já a Fig. 4 apresenta as curvas de taxa de erro de símbolos para uma freqüência Doppler normalizada $f_{D} T=0,007$. Comparando as Figs. 2 e 4, vê-se claramente a influência da velocidade do terminal móvel no desempenho do sistema. Quanto maior a velocidade, mais rápidas são as variações do canal e mais difícil se torna o rastreamento dos coeficientes do canal. Diferentemente do caso em que $f_{D} T=0,001$, o desempenho do receptor proposto sofre uma pequena degradação em relação ao receptor ótimo para $f_{D} T=0,007$. Para ambos os valores de $p_{T_{x}}$ simulados, o receptor semi-cego com o FK de complexidade reduzida fica a aproximadamente $1,5 \mathrm{~dB}$ do receptor com conhecimento perfeito do canal para uma taxa de erro de $10^{-3}$. Mais uma vez, os resultados indicam que o estimador proposto é robusto em relação à correlação espacial entre as antenas de transmissão. Isto pode ser verificado na Fig. 5, que apresenta o erro quadrático médio de estimação para alguns valores de $p_{T_{x}}$. Nesta figura, fica evidente que o desempenho do FK não é afetado pela correlação espacial entre as antenas. Comparando as Figs. 3 e 5, notamos que a ve-

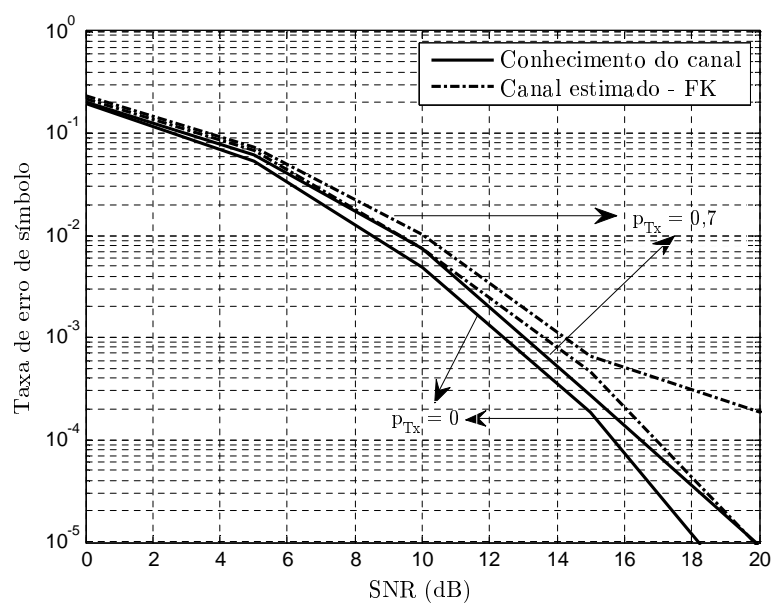

Fig. 4. Taxa de erro de símbolos para $f_{D} T=0,007$.

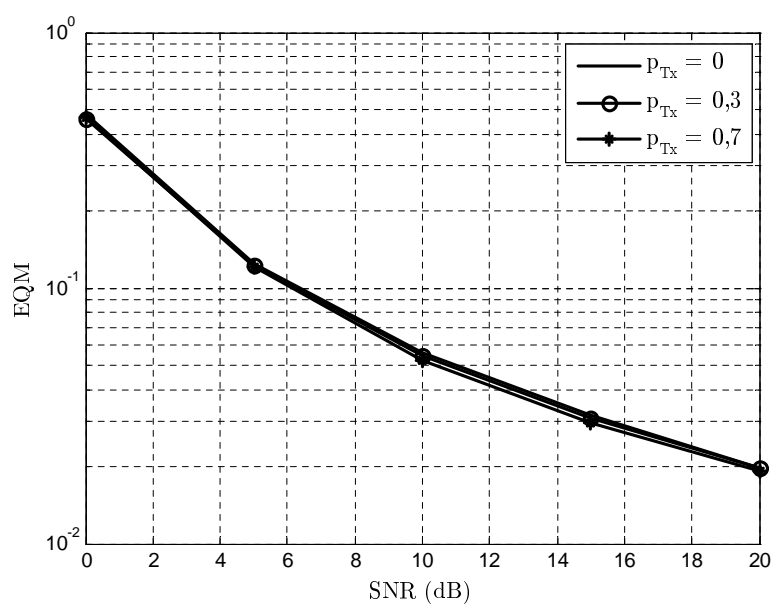

Fig. 5. Erro quadrático médio de estimação para $f_{D} T=0,007$.

locidade das variações temporais do canal tem uma influência muito maior que a correlação espacial no desempenho do FK com complexidade reduzida.

\section{Conclusões}

Neste artigo foi proposto um estimador de canal semicego, com complexidade reduzida, para sistemas MIMO empregando códigos espaço-temporais ortogonais por blocos. O algoritmo desenvolvido, baseado no filtro de Kalman, é capaz de estimar canais planos, variantes no tempo e com correlação espacial entre as antenas transmissoras e/ou receptoras. Antes de desenvolver o estimador de canal, no entanto, foi necessário estabelecer um modelo em espaço de estados para canais correlacionados e variantes no tempo. Resultados de simulações indicam que o estimador de canal proposto é robusto em relação à correlação espacial entre as antenas.

\section{AgRADECIMENTOS}

Os autores gostariam de agradecer à Fundação de Amparo a Pesquisa do Estado de São Paulo, FAPESP, e ao Conselho Nacional de Desenvolvimento Científico e Tecnológico, CNPq, pelo apoio financeiro. 


\section{REFERÊNCIAS}

[1] S. M. Alamouti, "A Simple Transmit Diversity Technique for Wireless Communications," IEEE Journal on Selected Areas in Communications, vol. 16, $\mathrm{n}^{\circ}$. 10, pags. 1451-1458, outubro 1998 .

[2] V. Tarokh, H. Jafarkhani, e A. R. Calderbank, "Space-Time Block Codes from Orthogonal Designs," IEEE Transactions on Information Theory, vol. $45, \mathrm{n}^{\circ} .5$, pags. 1456-1467, julho 1999.

[3] B. Vucetic e J. Yuan, Space-Time Coding. John Wiley and Sons, 2003.

[4] E. Larsson, P. Stoica, e J. Li, "Orthogonal Space-Time Block Codes: Maximum Likelihood Detection for Unknown Channels and Unstructured Interferences," IEEE Transactions on Signal Processing, vol. 51, $\mathrm{n}^{\circ}$. 2, pags. 362-372, fevereiro 2003.

[5] S. M. Kay, Fundamentals of Statistical Signal Processing - Estimation Theory. Prentice Hall, 1998, vol. 1.

[6] Z. Liu, X. Ma, e G. B. Giannakis, "Space-Time Coding and Kalman Filtering for Time-Selective Fading Channels," IEEE Transactions on Communications, vol. 50, $\mathrm{n}^{\circ}$. 2, pags. 183-186, fevereiro 2002.

[7] B. Balakumar, S. Shahbazpanahi, e T. Kirubarajan, "Joint MIMO Channel Tracking and Symbol Decoding Using Kalman Filtering," IEEE Transactions on Signal Processing, vol. 55, $\mathrm{n}^{\circ}$. 12, pags. 5873-5879, dezembro 2007.
[8] W. C. Jakes, Microwave Mobile Communications. New York: John Wiley and Sons, 1974.

[9] C. Komninakis, C. Fragouli, A. H. Sayed, e R. D. Wesel, "Multi-Input Multi-Output Fading Channel Tracking and Equalization Using Kalman Estimation," IEEE Transactions on Signal Processing, vol. 50, $\mathrm{n}^{\circ}$. 5, pags. 1065-1076, May 2002.

[10] E. Karami e M. Shiva, "Blind Multi-Input Multi-Output Channel Tracking Using Decision-Directed Maximum-Likelihood Estimation," IEEE Transactions on Vehicular Technology, vol. 56, $\mathrm{n}^{\circ}$. 3, pags. 1447-1454, May 2007.

[11] W. Weichselberger, M. Herdin, H. Özcelik, e E. Bonek, "A Stochastic MIMO Channel Model with Joint Correlation of Both Link Ends," IEEE Transactions on Wireless Communications, vol. 5, $\mathrm{n}^{\circ}$. 1, pags. 90-100, janeiro 2006.

[12] M. Enescu, T. Roman, e V. Koivunen, "State-Space Approach to Spatially Correlated MIMO OFDM Channel Estimation," Signal Processing, vol. $87, \mathrm{n}^{\circ} .9$, pags. 2272-2279, setembro 2007.

[13] G. H. Golub e C. F. V. Loan, Matrix Computations, $3^{\mathrm{a}}$ ed. John Hopkins University Press, 1996. 